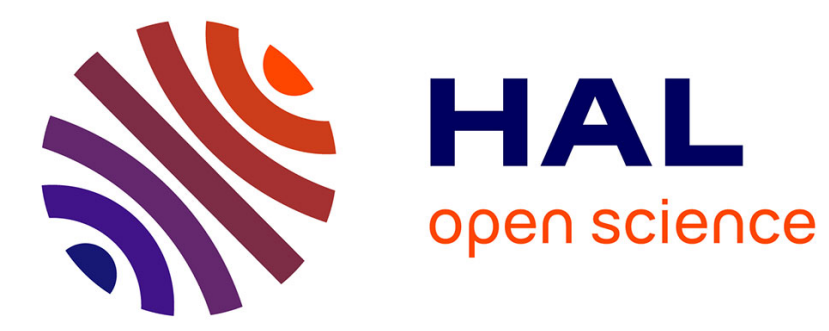

\title{
Scaling laws and intermittency in phase turbulence
}

Stéphane Zaleski, P. Lallemand

\section{To cite this version:}

Stéphane Zaleski, P. Lallemand. Scaling laws and intermittency in phase turbulence. Journal de Physique Lettres, 1985, 46 (17), pp.793-796. 10.1051/jphyslet:019850046017079300 . jpa-00232900

\section{HAL Id: jpa-00232900 https://hal.science/jpa-00232900}

Submitted on 1 Jan 1985

HAL is a multi-disciplinary open access archive for the deposit and dissemination of scientific research documents, whether they are published or not. The documents may come from teaching and research institutions in France or abroad, or from public or private research centers.
L'archive ouverte pluridisciplinaire HAL, est destinée au dépôt et à la diffusion de documents scientifiques de niveau recherche, publiés ou non, émanant des établissements d'enseignement et de recherche français ou étrangers, des laboratoires publics ou privés. 
Classification

Physics Abstracts

$05.40-47.25$

\title{
Scaling laws and intermittency in phase turbulence
}

\author{
S. Zaleski and P. Lallemand \\ Laboratoire de Physique de l'Ecole Normale Supérieure, 24 rue Lhomond, 75231 Paris Cedex 05, \\ France
}

(Reçu le 22 mars 1985, accepté sous forme définitive le 4 juillet 1985)

\begin{abstract}
Résumé. - Nous examinons numériquement certaines propriétés statistiques de l'équation de Kuramoto-Sivashinsky-Tsuzuki [1-3], décrivant des systèmes étendus près du point de transition vers la turbulence, à travers leurs fluctuations de phase. Le modèle ainsi constitué possède certaines analogies avec la turbulence hydrodynamique [4]. Une loi d'échelle surprenante y a été trouvée pour les fluctuations temporelles, que l'on peut interpréter comme un phénomène de diffusion anormale de la phase. Par ailleurs, certains autres résultats inattendus peuvent être interprétés comme des phénomènes d'intermittence.
\end{abstract}

\begin{abstract}
We investigate numerically the statistical properties of the Kuramoto-SivashinskyTsuzuki model [1-3], which describes the phase fluctuations of extended systems near the transition to turbulence. This model has some similarities with hydrodynamic turbulence [4]. The time fluctuations exhibit an unexpected scaling corresponding to anomalous diffusion of the phase. Other results also point to an intermittent behaviour.
\end{abstract}

\section{Phase turbulence as hydrodynamics with many chaotic degrees of freedom.}

Many pattern-forming instabilities can be modelled by the equation :

$$
\phi_{t}+\phi_{x x}+\phi_{x x x x}+\phi_{x}^{2}=0, \quad 0<x<L,
$$

where subscripts stand for derivatives. It is shown to describe the temporal phase of coupled chemical oscillators [1]. It also describes the behaviour of other extended pattern forming systems, such as flame fronts [2] and fluid interfaces [3]. The derivative of the phase satisfies an equation of hydrodynamical type :

$$
v_{t}+v_{x x}+v_{x x x x}+2 v v_{x}=0, \quad 0<x<L .
$$

where $v=\phi_{x}$. In this form the model is reminiscent of the Burgers equation which was also proposed as a model for turbulence. But in contrast with the Burgers equation, (1) displays a sustained chaotic behaviour $[4,5]$. This occurs for large enough values of $L$ and for the boundary conditions

$$
v=v_{x}=0, \quad x=0, L
$$


The chaos then involves a number of degrees of freedom proportional to $L$. Rigorous bounds on this number were given for even periodic solutions [6]. The model (2) somehow mimics hydrodynamical turbulence where a chaotic behaviour with a large number of degrees of freedom is also expected [7]. It would hence be interesting to know whether statistical theories of turbulence can be applied in this case. A detailed check of such theories is made possible by modern computing facilities. The analogy with hydrodynamics is relevant because, as pointed out by several authors, basic ideas about turbulence, like the Kolmogorov theory of energy cascade [8], depend very little on the accurate form of the Navier-Stokes equations. Actually, the Kolmogorov theory mainly relies on the existence of a quantity approximately conserved in the equations, the kinetic energy. In equation (2), the quantity $v^{2}$ might play a similar rôle. However, it has been shown that no inertial range cascade existed for $(2)[4,9]$. There seems to be, however, another scaling in the space spectrum : a range of equipartition for the "energy " $v^{2}$ has been found numerically [4] and using the D.I.A. perturbation method [10-11].

Further pursuing the above analogy, we note that $v$ is exactly conserved, just as the actual momentum in hydrodynamics. "Exact conservation " means that there exists a current $J(x, t)$ such that

with

$$
v_{t}+J_{x}=0 \text {, }
$$

$$
J=v_{x}+v_{x x x}+v^{2}
$$

Another interesting quantity is

$$
Q(t)=\int_{0}^{L} v(x, t) \mathrm{d} x
$$

$Q$ can fluctuate only through fluctuations of $J$ :

$$
Q_{t}=J(0)-J(L) \text {. }
$$

The conservation law (4), and the related similarity with hydrodynamics motivated the numerical study of the statistical properties of $v, J$ and $Q$.

\section{Numerical results.}

Non linearities favour the existence of a rightward oriented mean current. Integrating numerically equations (2) and (3), we measured $\langle J(0)\rangle=0.4$. Simple arguments can relate this current to the time-average $\langle v(x)\rangle$ of the solution (Fig. 1) (here and thereafter, we refer to numerical

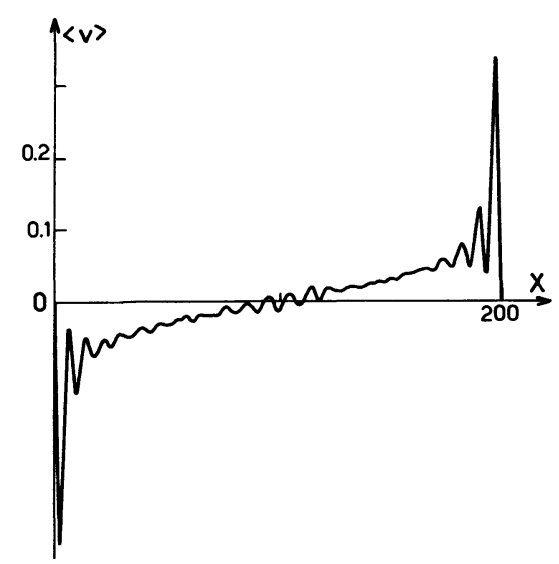

Fig. 1. - Mean value of $v(x)$ for $L=200$. Oscillations in the middle may be due to imperfect averaging. 
time averages and we assume that they are equivalent to probabilistic averages). It is seen that the quantity $v$ accumulates near the right boundary and is depleted near the left one. A mean slope is seen near the centre. When the length $L$ is increased, fluctuations are increasingly uniform in space, but the humps near the boundaries remain.

Fluctuations of $Q$ will arise when $J$ departs from its mean value. In hydrodynamic turbulence, the statistical analysis involves the study of structure functions of the type :

$$
\left\langle|v(x+l)-v(x)|^{p}\right\rangle \simeq C_{p} l^{h-\xi_{p}}
$$

where $h$ is the self-similarity exponent and $\xi_{p}$ are intermittency corrections. For (1), a quantity of interest is

$$
\chi_{n}(\tau)=\left\langle(Q(t+\tau)-Q(t))^{2 n}\right\rangle .
$$

The simplest case would be a Brownian motion for $Q$, with the scaling

$$
\chi_{n}=C_{n} \tau^{n}
$$

To emphasize the connection with hydrodynamic turbulence, we present another heuristic argument. In the following reasonning, we do not pretend to be rigorous, but to show the possible relationship of our results to existing statistical theories. Simple theories of turbulence argue that the space averaged velocity $V$ should obey a Langevin equation of the form :

$$
V_{t}=v_{\text {eff }} \Delta V+f
$$

where $v_{\text {eff }}$ is an effective viscosity, or turbulent eddy diffusivity, and $f$ is a Markovian white noise. A positive effective viscosity $v$ has also been predicted for equation (1) [11]. Let us examine the relaxation of fluctuations of $Q$. From (10) with appropriate boundary conditions one would get :

$$
\langle Q(t+\tau) Q(t)\rangle=\mathrm{e}^{-v a \tau}\left\langle Q^{2}\right\rangle
$$

where $a$ is the smallest eigenvalue of the diffusion operator on $(0, L)$. Our numerical results show instead that above very short time increments, of the order of 8 , one has the scaling

$$
\chi_{n}=C_{n} \tau^{\mu n} .
$$

In all our simulations one has $0.57<\mu<0.62$. The scaling (11) is observed over two decades, after which the increment $\chi_{n}$ begins to saturate. The saturation time increases with $L$. The linear growth of the exponents $n \mu$ was observed up to $n=6$. This proportionality of the exponent to $n$ is very accurately verified (Fig. 2).

These results have been obtained by averaging over very long times and with rather wide length $L$. The finite difference scheme of [4] and [5] was used with grid spacing down to 0.1 and time steps larger or equal to 0.05 . No significant variation of $\mu$ was recorded when space and time resolution was increased. Computations were made up to $T=2 \times 10^{5}$, and for several lengths between 100 and 500 , showing no significant dependency on $L$. This is reasonable, since a general property of the model is that most statistical properties reach an asymptotic regime for $L$ above 150. In particular, this is the length for which the spectrum of Liapounov exponents reaches its asymptotic shape [5].

It is already known [9] that statistical properties like the flatness of the $m$-th time derivative $F_{m}=\left\langle v^{(m) 4}\right\rangle\left\langle\left\langle v^{(m) 2}\right\rangle^{2}\right.$ have different values close to the boundaries and in the bulk. (In the bulk, $F_{m}$ is constant, up to statistical error). Thus (11) might be related to special characteristics of flux at the boundaries. To test this, we measured $Q_{1}(t)=\int_{L / 4}^{3 L / 4} v(x, t) \mathrm{d} x$, for $L=400$. We 


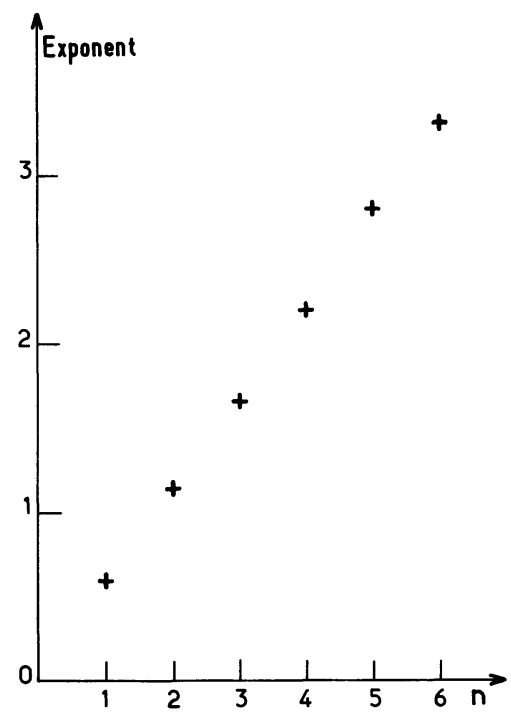

Fig. 2. - Values of the exponents for the moments $\chi_{n}$ (see text).

find again the law (11). This motivates the conjecture that (11) does not depend on boundary conditions. We return to this point below. We also looked at a local quantity like $\langle(v(x, t+\tau)-$ $v(x, t))^{2}>$ for some $x$ in $(0, L)$. This quantity, however, does not display the scaling behaviour (11).

To our knowledge, there is no explanation, at present, of the law (11). In particular, we found no similarity argument leading to such a law. We however looked at other statistical properties that might be related to this scaling. In particular, a slow diffusion might be related to form of intermittency [12]. In the case $n=2$, the scaling law (11) is equivalent to a slope $\alpha=1+\mu$ for the frequency spectrum, to be compared to the value $\alpha=1.75$ found in the literature [12]. The corresponding simulations were made for $L=64$, a length for which $\mu$ is larger than its asymptotic value. A statistical study of the quantity $Q$ showed that it is Gaussian-distributed.

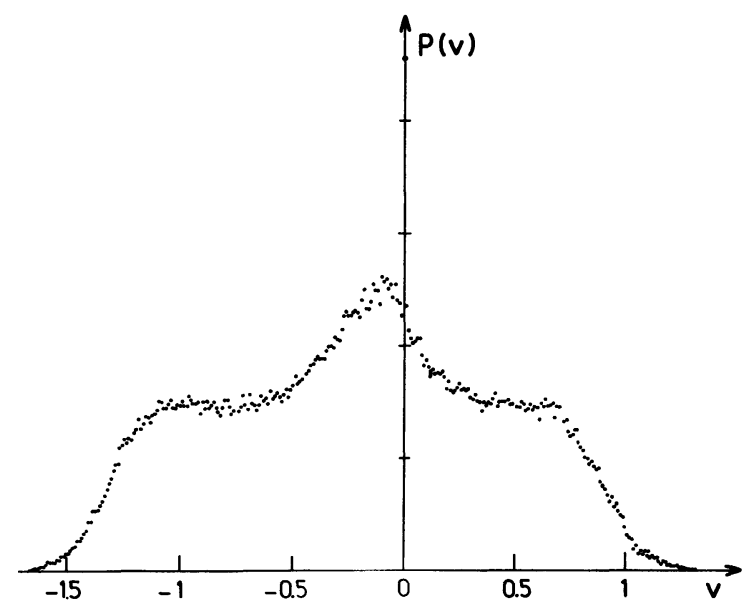

Fig. 3. - Histogram of values of $v(x, t)$ for $x=L / 10$. The histogram is obtained with increments of $v$ of 0.01 . The histogram of $v(9 L / 10, t)$ is symmetric to the present one within statistical error. 
However when studying time derivatives of $Q$, one finds that the moments of order of 4 and 6 increase very fast with the derivation order pointing to the intermittent character of the time fluctuations of $Q$. Another striking phenomenon is the shape of probability density of $v$ for fixed $x$ (Fig. 3). It has already been shown that the fluctuations of $v$ are sub-Gaussian [9]. This is reflected in the rapid damping of $P(v)$ at large values of $v$. The existence of a delta function-like peak for $v=0$ points to a form of intermittency for $v$ itself. The recurrence of the unstable solution $v(x, t)=0$ might explain this peak, which is not observed when there is only a small number of degrees of freedom $N$.

\section{Conclusion.}

We found an anomalous scaling for the space averaged momentum $Q$, related to a form of intermittency. An important point, not yet stressed, is that $Q$ is the phase increment through $(0, L): Q=\phi(L)-\phi(0)$. Thus anomalous diffusion of $Q$ is an anomalous diffusion of the phase, a quantity that can be measured experimentally [13]. It might also be relevant to make a remark on a somehow different problem. (1) can be studied with periodic boundary conditions. The solutions can then be apparently chaotic for a very long time and then relax suddenly to stationary solutions. This was observed by several people [14]. It would be interesting to know whether the time spent by the system to find the stationary solution is related to the anomalous diffusion of the phase. But for the time being, the statistics of phase chaos remain remarkably mysterious.

\section{Acknowledgments.}

We wish to thank Yves Pomeau for suggesting the subject of this investigation and for very valuable advice. We used a grant of CRAY time of the CCVR (Palaiseau), and a FPS 164 computer with financing from the DRET (Paris), and acknowledge those institutions for their support. One of us (S.Z.) also benefited from a visit to CNLS at the Los Alamos National Laboratory.

\section{References}

[1] Kuramoto, Y. et Tsuzuki, T., Prog. Theor. Phys. 55 (1976) 356.

[2] Sivashinsky, G. I., Acta Astron. 4 (1977) 1177.

[3] Sivashinsky, G. I. and Michelson, D. M., Prog. Theor. Phys. 63(1980) 2112.

[4] Pomeau, Y., Pumir, A. and Pelce, P., J. Stat. Phys. 37 (1984) 39.

[5] Manneville, P., "Liapounov exponents for the Kuramoto Sivashinsky equation ", to appear in the proceedings of the INRIA-NSF conference on Macroscopic modeling of turbulent flow, Nice, France, 10-14 December 1984.

[6] Nicolaenko, B., Teman, R. and Scheurer, B., Physica D, to appear.

[7] Pomeau, Y. and Zaleski, S., The Kuramoto Sivashinsky equation : a caricature of hydrodynamic turbulence ?, to appear in the proceedings of the INRIA-NSF conference on Macroscopic modeling of turbulent flow, Nice, France, 10-14 December 1984.

[8] Monin, A. S. and Yaglom, A. M., Statistical Fluid Mechanics (MIT press) 1975.

[9] Pumir, A., J. Physique 46 (1985) 511.

[10] Fujisaka, H. and Yamada, T., Prog. Theor. Phys. 57 (1977) 734.

[11] Yакнот, V., Phys. Rev. A 24 (1981) 642.

[12] Manneville, P., Phys. Lett. A 84 (1981) 129.

[13] See for instance FAUVE, S., Thèse d'Etat, Paris 1984. In this case $J=\phi t$ is a frequency.

[14] MANNEVILLE, P., private communication and SCHEURER, B., private communication. 